\title{
Coupled surface plasmon resonance on gold nanocubes - investigation by simulation
}

\author{
Attila Bonyár \\ Department of Electronics Technology \\ Budapest University of Technology and Economics \\ Budapest, Hungary \\ bonyar@ett.bme.hu
}

\author{
Géza Szántó, István Csarnovics \\ Department of Experimental Physics \\ University of Debrecen \\ Debrecen, Hungary
}

\begin{abstract}
The refractive index sensitivity of coupled plasmonic nanostructures, namely gold nanocubes in various arrangements, were simulated with the MNPBEM Matlab toolbox. The size of the cubes, the distance between the particles were the running parameters. It was found that the enhancement factor (which characterize the increase in the peak shift for multiparticle arrangements compared to single-particle models) is an exponential function of $(D / a)$ where $D$ is the gap between the particles and $a$ is the edge length of the cube. It was also found that significant plasmonic coupling effects starts below $0.5 \mathrm{D} / \mathrm{a}$ for cubical nanoparticles.
\end{abstract}

\section{Keywords-plasmonics, nanocubes, refractive index}

\section{INTRODUCTION}

The refractive index sensitivity of localized surface plasmon resonance (LSPR) based sensors is depending on several parameters, including the material type, size and shape of the particle and also the spatial arrangement of multi-particle systems [1]. It is already proven that with the proper nanostructures in the proper arrangement the sensitivity of LSPR (considering molecular or biosensing applications) can reach the sensitivity of classic Kretschmann-configuration based SPR devices on the market [2]. The general aim of our research group is the development of technologies, which would enable the cost-effective fabrication of highly ordered nanoparticle systems on large surface areas (several $\mathrm{cm}^{2}$ ). Hence, one motivation of the presented simulations is to study the connection between the particle size/shape/arrangement and the bulk refractive index sensitivity of the nanoparticle based sensor, by using gold nanocubes in this particular case.

The MNPBEM Matlab toolbox, which utilizes the boundary element method (BEM) approach, provides a convenient way for the simulation of coupled plasmonic nanostructures [3]. Besides the advantageous short running times compared to other finite element methods, it also enables the relatively simple inclusion of substrates in the model [4].

\section{Modeling AND Simulation PARAMETERS}

Although in this work only gold nanospheres are investigated, the MNPBEM Toolbox supports the convenient setting up of particles with elementary shape (sphere, rod, torus, and cube). The investigated nanocube arrangements were created with the 'tricube' function and are illustrated in Fig. 1.
The changing parameters were the edge of the nanocubes $(a)$ and the distance between the particles $(D)$. A plane wave excitation was used with light propagation in the $\mathrm{Z}$ and light polarization in the $\mathrm{X}$ directions (see Fig. 1). For the evaluation of the plasmonic behavior of the particles the resulting extinction cross sections were used. To calculate the bulk refractive index sensitivity, the media surrounding the nanoparticles was changed between air $(n=1)$ and water $(n=1.33)$. The sensitivity of a single particle $(S,[\mathrm{~nm} / \mathrm{RIU}])$ is defined as the shift of the extinction peak divided by the refractive index change of the media. For multi-particle arrangements the enhancement factor is defined as the absolute peak shift of the multi-particle arrangement (due to refractive index change) divided by the absolute peak shift of the single particle model. In this way the enhancement factor quantify the increased peak shift which originates from the interparticle coupling effects, compared to the single particle model. In this current work, only simple, twoparticle arrangements are investigated.

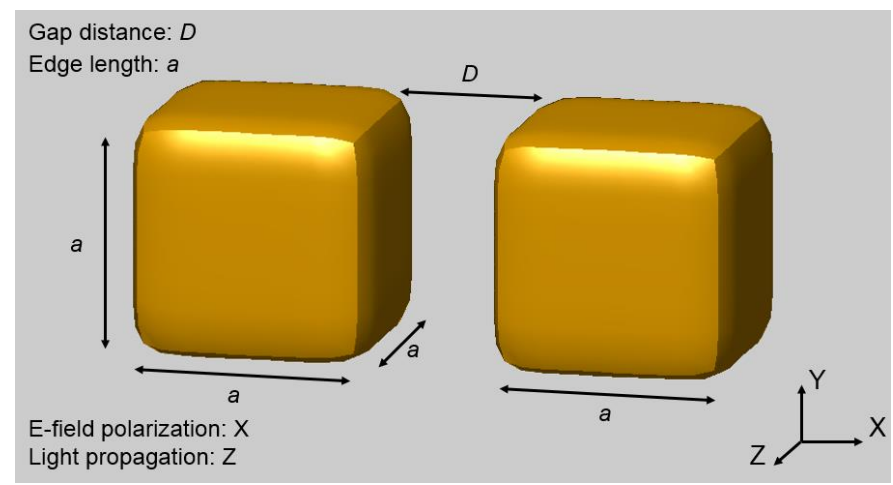

Fig. 1. Illustration of the investigated gold nanocube arrangement. $D$ : gap distance between the two nanocubes, $a$ : edge length.

Two dielectric functions with tabulated values are available for the plasmonic simulation of gold particles, based on the optical constants of Johnson and Palik, respectively. Although the peak shift (between air and water) obtained with the constants are nearly the same, the Palik dielectric function yields systematically shorter peak wavelengths, and also secondary peaks could be observed, which increase significantly when the distance between particles is decreased in multi-particle arrangements (data not shown). Taking these observations into consideration, the Johnson dielectric function was used for further calculations. 


\section{RESULTS AND DISCUSSION}

\section{A. Solver optimization}

The solver of the MNPBEM Matlab Toolbox offers two simulation approaches for the investigation of our nanocube arrangements. Using 'Retarded simulation', the full Maxwell equations are solved on the arrangement. Although this approach can be considered as more precise it is also more time consuming than the 'Quasistatic simulation' which is developed to provide a faster approach. Although the developers claim that the quasistatic approach usually give accurate and reliable results for nanoparticles below $100 \mathrm{~nm}$, they also encourage users to compare the two solvers on their particular problem.

Comparing the extinction cross section spectra obtained by the retarded and quasistatic solvers, the most notable difference is the appearance of smaller, secondary peaks in the spectra obtained by retarded simulations. As can be seen in the results presented in Fig. 2 and 3 the appearance and extent of these secondary peaks is also depending on the size and distance between the nanocubes, on the grid density (the number of mesh divisions per cube edge) and also, on the refractive index of the media.

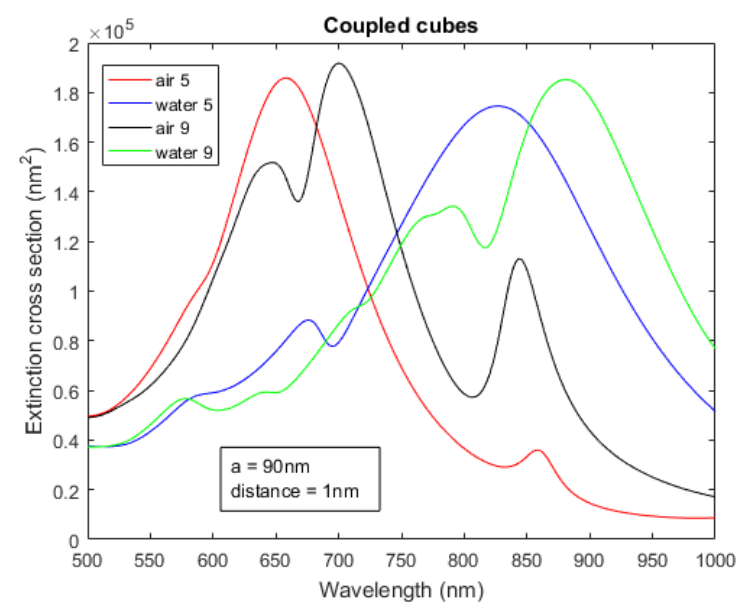

Fig. 2. Extinction cross section spectra of nanocubes with $90 \mathrm{~nm}$ edges $(a)$ and $1 \mathrm{~nm}$ gap $(D)$ simulated in air and water environment with 5 and 9 grid density (divisions/edge).

Based on Fig. 2 and 3 it can be said, that decreasing the gap between the particles, increasing the size of the cube, the grid density or the refractive index of the media all increase the amount of secondary peaks in the spectra, which can possibly be related to the appearance of higher plasmonic resonance modes. A problem with the secondary peaks presented in Fig. 2 is that by scaling the gap distance between the particles it is possible that the dominant peak changes (dominance transition between multiple peaks) in function of the distance. By defining the sensitivity as the absolute difference between the dominant peaks (simulated in air and water), this abrupt change in dominant peak position can cause jumps in an otherwise continuous sensitivity/distance function, as can later be seen in Figs. 4, 5, 6 .

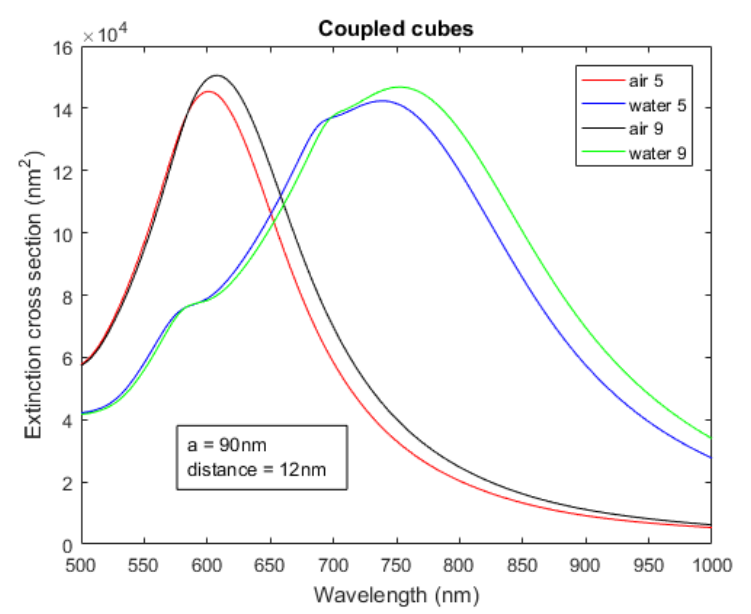

Fig. 3. Extinction cross section spectra of nanocubes with $90 \mathrm{~nm}$ edges $(a)$ and $12 \mathrm{~nm}$ gap $(D)$ simulated in air and water environment with 5 and 9 grid density (divisions/edge).

We have to note, that although it is possible to observe small secondary peaks in the case of the quasistatic solver as well (especially at small gap distances), they are usually too small compared to the dominant peak to be considered as problems. It can also be seen in Figs. 2 and 3 that increasing the grid density from 5 to 9 increased the obtained refractive index sensitivities (the absolute difference between the peaks in water and air) to a small extent. Since denser grids significantly increase the running time with the more time-consuming retarded simulations, 9 divisions/cube edge was used in the following investigations.

\section{B. Dependence of the enhancement factor on $\mathrm{D} / \mathrm{a}$}

Fig. 4 presents the obtained enhancement factor values in function of the gap distance between the nanocubes, for cube edge lengths from 10 to $90 \mathrm{~nm}$. The results of the retarded and quasistatic solvers are also compared in this figure. The jumps which can be observed below $10 \mathrm{~nm}$ for the results obtained with the retarded solver can be attributed to the discussed transition of the dominant peak between multiple peaks in an otherwise exponential distance-dependence.

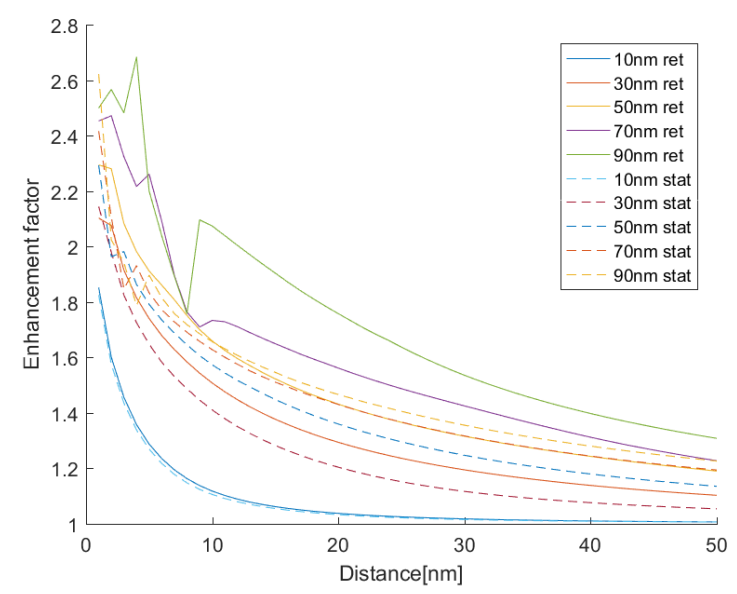

Fig. 4. Dependence of the enhancement factor on the distance between the nanocubes, for the retarded and quasistatic solvers. 
The difference between the retarded and quasistatic solvers is more pronounced for the larger nanocubes, where the retarded solver yields significantly higher enhancement factors. Fig. 5 illustrates the same results in a dimensionless way, in the function of $D / a$. It is interesting to note that in this representation, by using the quasistatic solver, the enhancement factor curves decrease by increasing the cube size, while with the retarded solver it increases for larger cubes. Based on the results it can be stated, that in order to achieve a significant field enhancement effect and enhanced sensitivity compared to the single-particle model, the nanocubes should be very close to each other, specifically, on a relative scale of distance/edge length $(D / a)$ the significant enhancement starts below 0.5 . If the distance between the particles is substantially large (e.g. $D / a>0.5)$, the results converge into the theoretical shift for single-particles (enhancement factor $=1$ ).

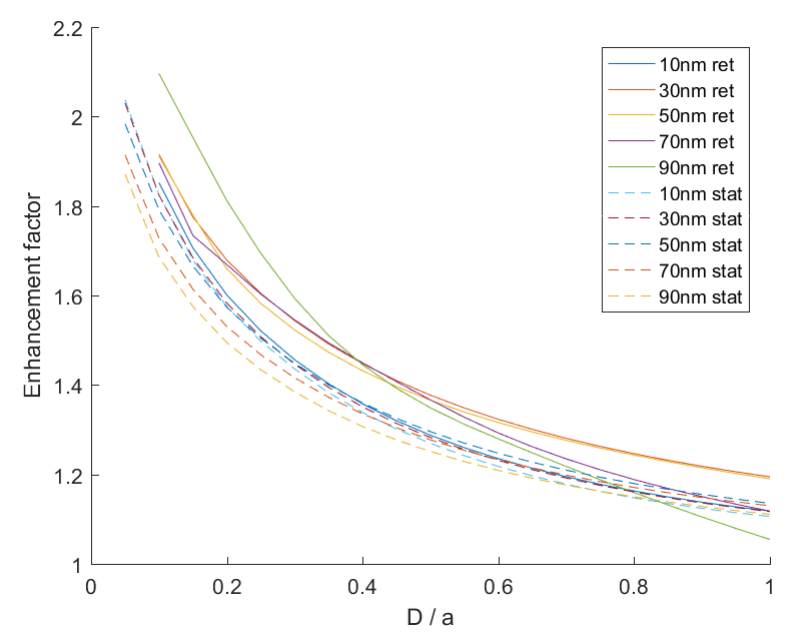

Fig. 5. Dependence of the enhancement factor on $D / a$ for the retarded and quasistatic solvers.

\section{Comparison with nanospheres}

Fig. 6 compares the results simulated on the nanocubes with previous simulation results obtained by using spherical nanoparticles. The paired nanospheres were simulated with the exact same parameters, by using the gap distance $(D)$ and the particle diameter $\left(D_{0}\right)$ as equivalent running parameters. It can be seen, that there is also a significant difference between the retarded and quasistatic simulations for nanospheres as well, along with the marks of dominant peak transition. The results indicate, that although the enhancement starts earlier (by decreasing $D / a$ ) for the nanocubes, below $0.3-0.2 D / D_{0}$ the spherical particles have significantly higher enhancement. The obtained enhancement factor curves can be approximated as exponential functions of the gap between the particles. This exponential decay is in good agreement with the theory of quantum tunneling of photons through the gap during evanescent coupling, and also with previous experimental results [5]. The decay length is depending on the shape of the particles, both on the opposing surface areas and on the curvature of the particles. The investigation and generalization of the effect of nanoparticle shape/area on the enhancement factor will the next step in our work.

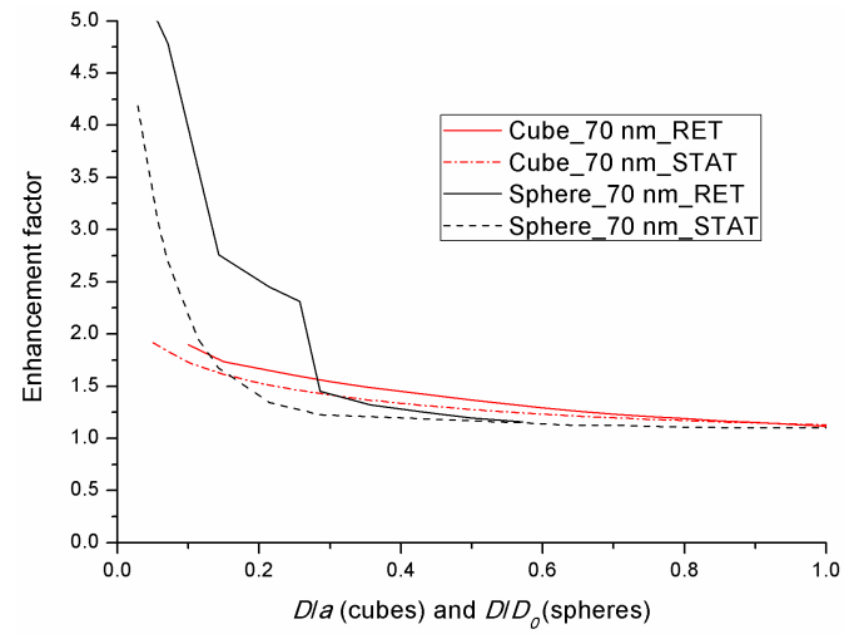

Fig. 6. Dependence of the enhancement factor on $D / a$ (for nanocubes) and $D / D_{0}$ (for nanospheres) for the for the retarded and quasistatic solvers.

\section{CONCLUSIONS}

The effect of size and gap distance on the plasmonic behavior (especially plasmonic coupling) of paired nanocubes was investigated through simulation with the MNPBEM Matlab toolbox. Calculations with both the retarded and quasistatic solvers of the toolbox showed, that the enhancement factor (which characterize the increase in the peak shift for multiparticle arrangements compared to single-particle models) is an exponential function of $(D / a)$ where $D$ is the gap between the cubes and $a$ is the length of the edges. By comparing the results with simulations made on spherical nanoparticles we found that spheres have significantly (2-3 times) higher enhancement factor below $0.2-03 . D / D_{0}$ (where $D_{0}$ is the particle diameter), compared to the cubes.

\section{ACKNOWLEDGMENT}

This research was supported by the European Union and the State of Hungary, co-financed by the European Social Fund in the framework of TÁMOP 4.2.4. A/2-11-1-2012-0001 'National Excellence Program'. Attila Bonyár is grateful for the support of the János Bolyai Research Scholarship of the Hungarian Academy of Sciences.

\section{REFERENCES}

[1] M.H. Tu, T. Sun, K.T.V. Grattan, "LSPR optical fibre sensors based on hollow gold nanostructures" Sensors and Actuators B 191, 37-44. 2014

[2] A. Dimitrev, Nanoplasmonic Sensors, Springer, ISBN 978-1-4614-3932$5,2012$.

[3] U. Hohenester, A. Trügler, "MNPBEM - A Matlab toolbox for the simulation of plasmonic nanoparticles" Computer Physics Communications 183, 370-381, 2012.

[4] J. Waxenegger, A. Trügler, U. Hohenester, "Plasmonics simulations with the MNPBEM toolbox: Consideration of substrates and layer structures" Computer Physics Communications 193, 138-150, 2015.

[5] A. Bonyár, "Simulation of the refractive index sensitivity of coupled plasmonic nanostructures", Procedia Engineering, in press, 2016.

[6] K.-H. Su, Q.-H. Wei, and X. Zhang, "Interparticle Coupling Effects on Plasmon Resonances of Nanogold Particles" Nano Letters 3(8), 10871090, 2003. 